\title{
Gold-free GaAs/GaAsSb heterostructure nanowires grown on silicon
}

\author{
S. Plissard, ${ }^{1, a)}$ K. A. Dick, ${ }^{2,3}$ X. Wallart, ${ }^{1}$ and P. Caroff ${ }^{1}$ \\ ${ }_{1}^{1}$ Institut d'Electronique, de Microélectronique et de Nanotechnologie, UMR CNRS 8520, Avenue Poincaré, \\ B.P. 60069, 59652 Villeneuve d'Ascq, France \\ ${ }^{2}$ Solid State Physics, Lund University, Box 118, S-22100 Lund, Sweden \\ ${ }^{3}$ Polymer and Materials Chemistry, Lund University, Box 124, S-22100 Lund, Sweden
}

(Received 9 February 2010; accepted 27 February 2010; published online 22 March 2010)

\begin{abstract}
Growth of GaAs/GaAsSb heterostructure nanowires on silicon without the need for gold seed particles is presented. A high vertical yield of GaAs nanowires is first obtained, and then $\mathrm{GaAs}_{\mathrm{x}} \mathrm{Sb}_{1-\mathrm{x}}$ segments are successfully grown axially in these nanowires. GaAsSb can also be integrated as a shell around the GaAs core. Finally, two GaAsSb segments are grown inside a GaAs nanowire and passivated using an $\mathrm{Al}_{\mathrm{x}} \mathrm{Ga}_{1-\mathrm{x}} \mathrm{As}$ shell. It is found that no stacking faults or twin planes occur in the GaAsSb segments. @ 2010 American Institute of Physics. [doi:10.1063/1.3367746]
\end{abstract}

Lattice-mismatched III-V semiconductor heterostructures are important for optoelectronic, nanoelectronic, and energy applications. Their integration on the advantageous $\mathrm{Si}$ platform is still facing major challenges, because of large lattice mismatches, polar/nonpolar growth, and differences in thermal expansion coefficients. Nanowires (NWs) have been considered as good candidates to overcome some of these issues and to facilitate the growth of III-V heterostructures at the nanoscale on silicon, thanks to the efficient strain relaxation at their free borders. ${ }^{1}$ However, gold, the most used seed particle for NW growth, is known to create detrimental midgap defects in silicon and should therefore be avoided in Si-compatible technological processes. Recently, the Fukui group $^{2}$ and Paek et al. ${ }^{3}$ reported direct growth on silicon, respectively, of InAs catalyst-free NWs arrays by metalorganic vapor-phase epitaxy (MOVPE), and self-catalyzed GaAs NWs by molecular beam epitaxy (MBE), thus opening a viable route for silicon integration of III-V devices. ${ }^{4}$ One of the most interesting materials for telecommunication or energy applications is $\mathrm{GaAs}_{\mathrm{x}} \mathrm{Sb}_{1-\mathrm{x}}$, as its wavelength can be tuned between 0.9 and $1.8 \mu \mathrm{m}$, and it allows type II band alignments with standard arsenide semiconductors. ${ }^{5}$ This possibility to extend band gap engineering to type II band alignments, which is interesting for separation of holes and electrons, ${ }^{6}$ could find applications in future solar cell designs. Gold-free growth of Sb-containing heterostructures has never been reported up to now by use of standard epitaxy systems.

In this paper, we present a study of axial and core-shell gold-free GaAs/GaAsSb NW heterostructures grown on native oxide-covered $\mathrm{Si}(111)$ substrates by gas source molecular beam epitaxy. Arsenic $\left(\mathrm{As}_{2}\right)$ is obtained by thermal cracking of arsine gas $\left(\mathrm{AsH}_{3}\right)$, a standard effusion cell is used for gallium and a valved-cracker cell is used for antimony $\left(\mathrm{Sb}_{2}\right)$. Growth rate and V/III ratios are calibrated using reflection high energy electron diffraction specular intensity oscillations. Lightly $\mathrm{n}$-doped Siltronix $\mathrm{Si}(111)$ substrates were loaded without any treatment in the MBE reactor, and the native oxide thickness was evaluated to be $10 \pm 1 \AA$ by angle-resolved $\mathrm{x}$-ray photoemission spectroscopy (XPS). The

\footnotetext{
${ }^{\text {a) }}$ Author to whom correspondence should be addressed. Electronic mail: sebastien.plissard@free.fr.
}

growth temperature was set at 630 or $650{ }^{\circ} \mathrm{C}$ for all samples, using a $\mathrm{As} / \mathrm{Ga}$ ratio of 1.5 (As pressure: $5.10^{-6}$ torr), at a two-dimensional (2D) equivalent GaAs growth rate of 1 ML/s. The morphology was studied by a Zeiss Supra scanning electron microscope (SEM) at $10 \mathrm{kV}$. Crystal structure characterization was performed using a JEOL-3000F field emission transmission electron microscope (TEM) operated at $300 \mathrm{kV}$ in conventional TEM (CTEM) mode and in highangle annular dark field (HAADF)-scanning TEM (STEM) mode. TEM images were recorded along the $\langle\overline{1} 10\rangle$ zone axis (cubic notation) and compositions were determined using $\mathrm{x}$-ray energy dispersive spectroscopy (EDS) operated in HAADF-STEM mode. The distribution of the present elements was studied by linescans and point composition spectra. The EDS linescans illustrate the heterostructure material changes qualitatively, whereas quantitative point scan analysis is used to extract precise compositions. In the following, all compositions are mean ones, and are given in atomic percents (at. \%). All presented TEM and EDS data was assessed to be representative of the whole NW samples by analyzing five to ten different NWs for each growth condition.

Figure 1(a) shows a SEM image (at $30^{\circ}$ tilt angle) of the typical GaAs NWs used in this study as a base/stem for the Sb-containing heterostructure NWs. The diameter of these hexagonally shaped NWs was typically in the range of 60 $100 \mathrm{~nm}$, and their length was found to be about $1 \mu \mathrm{m}$ long for a growth time of $5 \mathrm{~min}$. Most NWs grow in the $\langle 111\rangle$ direction perpendicular to the substrate, without measurable tapering. Some "bulk" material (i.e., GaAs crystallites forming a discontinuous layer) is present between the NWs, and

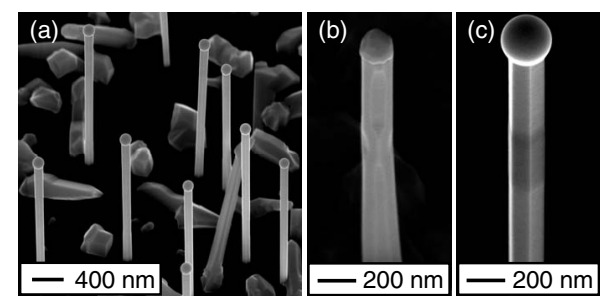

FIG. 1. SEM views at $30^{\circ}$ tilt angle of gold-free NWs grown on silicon. (a) GaAs NWs used as a base/stem of this study. (b) Core-shell GaAs/GaAsSb heterostructure. (c) Axial GaAsSb segment inserted in a GaAs NW. 


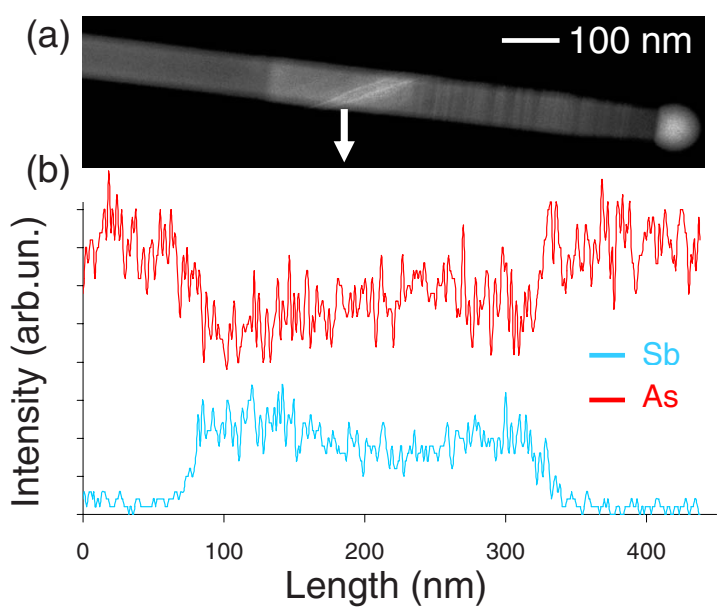

FIG. 2. (Color online) Characterization of an axial GaAsSb segment in a GaAs NW grown at $630{ }^{\circ} \mathrm{C}$. (a) HAADF STEM measurement of a GaAs wire with $\mathrm{a} \sim 270 \mathrm{~nm}$ long segment. (b) EDS linescan measurement of the wire in (a), qualitatively illustrating the $\mathrm{Sb}$ and As composition.

visible in Fig. 1(a). The composition of the nanoparticles measured by EDS is above 99.5 at. \% gallium. It shows, in agreement with similar studies by other groups, ${ }^{3,7}$ that the liquid gallium droplet is, in our case, the seed particle responsible for the enhancement of axial growth rate in the 〈111〉 direction leading to $\mathrm{NW}$ growth, in contrast to selective-area mechanisms reported by the Fukui group in the case of MOVPE grown NW arrays. ${ }^{8}$

Having established controlled parameters for gold-free GaAs NW growth on silicon, it is now possible to grow more advanced structures, like core-shell [Fig. 1(b)] or axial [Fig. $1(\mathrm{c})]$ heterostructures. In the first case, the growth of the NW is interrupted after the GaAs core, and by maintaining the arsenic flux on for 2 min with a closed Ga shutter, the gallium droplet is transformed into GaAs NW material, terminating axial NW growth. ${ }^{9}$ Then, a GaAsSb shell is created by opening simultaneously the $\mathrm{Ga}$ and $\mathrm{Sb}$ sources at the same temperature. Cross-sectional EDS analysis on these samples (not shown) indicates a GaAsSb shell, which seems fairly uniform although some samples present heterogeneous shell facets [Fig. 1(b)]. EDS point analysis suggests the shell thickness to be around $5 \mathrm{~nm}$, with $\mathrm{Sb}$ content around 11 at. $\%$

In the second case [Fig. 1(c)], a GaAsSb segment is grown axially during the GaAs NW growth by using a very high nominal antimony flux (equivalent to $3.5 \mathrm{ML} / \mathrm{s}$ ) to partially overcome the re-evaporation rate for this element, at the high growth temperature used. Two samples were grown, one at $630{ }^{\circ} \mathrm{C}$ (shown in Fig. 2), and one at $650{ }^{\circ} \mathrm{C}$ (only described in the text), in order to tune the antimony composition of the GaAsSb segment. Low magnification HAADFSTEM image of this segment is shown in Figs. 2(a) and 2(b) illustrates an EDS linescan profile along the length of the NW. A GaAsSb segment, $270 \mathrm{~nm}$ in length, is easily recognized when following the antimony signal in Fig. 2(b). Quantitative EDS point analysis of wires from this sample indicates a $\mathrm{Sb}$ content of 15 at. \%. Low antimony traces (around 2 at. \%) are also detected in the GaAs segment below the GaAsSb one, which suggests that a very thin $\mathrm{GaAsSb}$ shell forms in parallel to the dominant axial $\mathrm{GaAsSb}$ segment growth. A complementary growth of the same structure at $650^{\circ} \mathrm{C}$ confirms the temperature sensitiv-
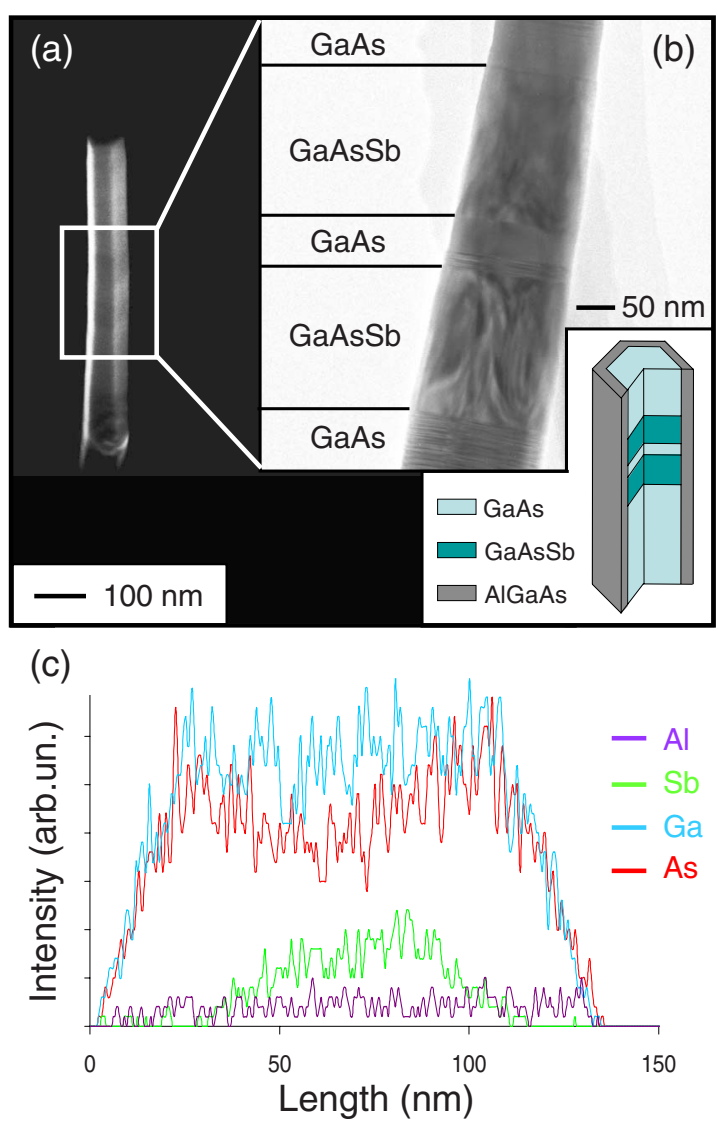

FIG. 3. (Color online) (a) SEM and (b) TEM images of GaAs NWs containing with 2 GaAsSb axial segments, and coated by an AlGaAs shell. The TEM image is taken along the $\langle\overline{1} 10\rangle$ zone axis. A schematic of the structure is also given. (c) Cross-sectional EDS measurement across a segment in a $\mathrm{NW}$, showing the $\mathrm{GaAs}_{\mathrm{x}} \mathrm{Sb}_{1-\mathrm{x}}$ core and the $\mathrm{Al}_{\mathrm{x}} \mathrm{Ga}_{1-\mathrm{x}} \mathrm{As}$ shell.

ity of the $\mathrm{Sb}$ incorporation with a measured $\mathrm{Sb}$ composition in the segment around 12 at. \%. The reduced average $\mathrm{Sb}$ content may be related to the higher competing evaporation of $\mathrm{Sb}$ at the higher temperature.

Having achieved single heterostructures, the following stage was to develop more complex structures. For that purpose, two GaAsSb segments were grown inside GaAs NWs, and then an AlGaAs shell was grown all-around. Growth time for these materials were $4 \mathrm{~min}$ (GaAs), $1 \mathrm{~min}$ (GaAsSb), $30 \mathrm{~s}$ (GaAs), 1 min (GaAsSb), and 2 min (GaAs). Finally a $1 \mathrm{~min} \mathrm{AlGaAs}$ shell was grown to passivate the whole structure against nonradiative recombination processes induced at the GaAs surface. ${ }^{3}$

Figures 3(a) and 3(b) show the SEM and TEM images of these NWs, along with a three-dimensional simple schematic of the nominal structure. Very interestingly, strain fields are visible in the TEM image only in the regions of the GaAsSb segments, allowing easy identification of these segments at low resolution. The appearance of strain fields can be explained by the following: an AlGaAs shell is approximately lattice-matched to GaAs for any aluminum content. However, if GaAsSb is elastically relaxed (one of the key advantages of NWs $){ }^{10}$ then only the GaAsSb/AlGaAs interfaces will be strained because of the AlGaAs shell covering the whole NW. A cross-sectional EDS linescan over a GaAsSb segment in one of these NWs is shown in Fig. 3(c). A $\sim 85 \mathrm{~nm} \mathrm{GaAsSb}$ core can be observed with a $\sim 25 \mathrm{~nm} \mathrm{Al}-$ GaAs shell all-around. Quantitative EDS point analysis indi- 


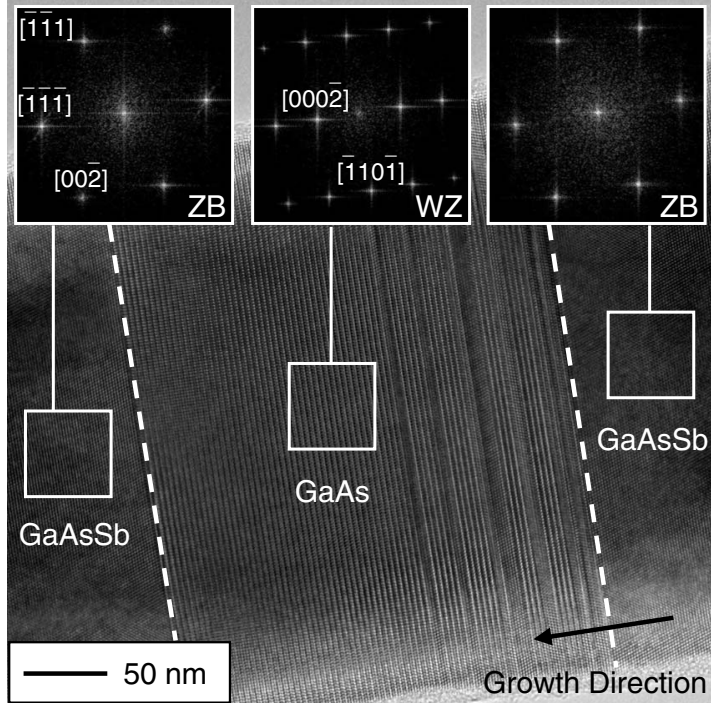

FIG. 4. HRTEM image of the axial GaAsSb/GaAs/GaAsSb heterostructure shown in Fig. 3, taken in the $\langle\overline{1} 10\rangle$ zone axis. Inset FFTs are given for each material, indicating the cubic (zinc blende) structure of the GaAsSb and hexagonal (wurtzite) structure of the GaAs in the center. Stacking faults are visible at the GaAsSb-GaAs interface but the switch back to GaAsSb is abrupt.

cated $\mathrm{Sb}$ composition in the segments around 17 at. \% (similar to the single axial heterostructure), and Al composition around 27 at. \%. The antimony compositions of the two segments are very similar and the aluminum composition of the shell is in good agreement with the 2D layer growth calibrations. As previously discussed for the single heterostructure sample, antimony traces are measured in the first two GaAs sections, while the top on the NW does not exhibit detectable antimony.

Finally, the crystal structure of these NWs was studied by high resolution TEM (HRTEM). The first GaAs segment typically exhibits stacking faults and wurtzite phase, as is often observed in gold-nucleated GaAs NWs. ${ }^{11}$ On the contrary, the GaAsSb segments exhibit perfect twin-free zinc blende phase. Figure 4 shows an HRTEM image of the two GaAsSb segments separated by GaAs. The crystal phase is also characterized in the fast Fourier transform (FFT) images added as insets. The control of phase purity in III-V NWs is a very active research area, as most of these naturally show polytypism. ${ }^{12}$ We note that our results are in perfect agreement with previously studied gold-seeded $\mathrm{GaSb}$ or $\mathrm{GaAsSb}$ NWs, ${ }^{13-15}$ although the growth mechanism, growth conditions, and substrate used are completely different. In view of these studies and of the present work, it is clear that this effect should be linked to the presence of antimony, independent of the nature of the seed particle droplet, and could be linked to its well known surfactant properties. ${ }^{16}$ Another interesting point is shown in Fig. 4, which shows the transition zone from the first (bottom) GaAsSb segment (right) to GaAs middle segment and second (top) GaAsSb segment. Both GaAsSb segments are perfect zinc blende but the middle GaAs segment has a wurtzite crystal structure. The transition from GaAsSb to GaAs on the right hand side shows some stacking faults, whereas the transition from GaAs to GaAsSb on the left hand side is abrupt. This could be due to $\mathrm{Sb}$ traces remaining in the particle after the switching sequence to GaAs is done.

In summary, we have reported successful growth of gold-free axial and lateral $\mathrm{GaAs} / \mathrm{GaAsSb}$ heterostructure NWs on silicon. Single GaAs/GaAsSb heterostructures were first shown. A $5 \mathrm{~nm}$ thick $\mathrm{GaAs}_{0.78} \mathrm{Sb}_{0.22}$ shell around a GaAs core and a $\sim 270 \mathrm{~nm} \mathrm{GaAs}{ }_{0.70} \mathrm{Sb}_{0.30}$ axial segment were then separately engineered. Finally, a complex structure composed of two axial $\mathrm{GaAs}_{0.76} \mathrm{Sb}_{0.34}$ segments in a GaAs NW, passivated with an $\mathrm{Al}_{0.54} \mathrm{Ga}_{0.46} \mathrm{As}$ shell, was detailed. The $\mathrm{GaAsSb}$ segments exhibit a perfect zinc blende structure, linked to the presence of antimony, while the GaAs contains significant wurtzite structure under the investigated growth conditions. The possibility to grow strained passivating AlGaAs shells is very promising for future microphotoluminescence studies of strained type II band-alignments in Sbcontaining III-V NW heterostructures.

The authors would like to acknowledge A. Addad, S. Godey, C. Coinon, and J.-L. Codron, respectively, for TEM characterizations, XPS analysis, and help with MBE work. Part of this work was funded by the Swedish Foundation for Strategic Research (SSF), the Swedish Research Council (VR), and the Knut and Alice Wallenberg Foundation.

${ }^{1}$ K. L. Kavanagh, Semicond. Sci. Technol. 25, 024006 (2010).

${ }^{2}$ K. Tomioka, J. Motohisa, S. Hara, and T. Fukui, Nano Lett. 8, 3475 (2008).

${ }^{3}$ J. H. Paek, T. Nishiwaki, M. Yamaguchi, and N. Sawaki, Phys. Status Solidi C 6, 1436 (2009).

${ }^{4}$ W. Wei, X. Bao, C. Soci, Y. Ding, Z. Wang, and D. Wang, Nano Lett. 9, 2926 (2009)

${ }^{5}$ D. L. Dheeraj, G. Patriarche, H. Zhou, T. B. Hoang, A. F. Moses, S. Grønsberg, A. T. J. van Helvoort, B. O. Fimland, and H. Weman, Nano Lett. 8, 4459 (2008).

${ }^{6}$ M. E. Pistol and C. Pryor, Phys. Rev. B 80, 035316 (2009).

${ }^{7}$ C. Colombo, D. Spirkoska, M. Frimmer, G. Abstreiter, and A. Fontcuberta i Morral, Phys. Rev. B 77, 155326 (2008).

${ }^{8}$ K. Ikejiri, J. Noborisaka, S. Hara, J. Motohisa, and T. Fukui, J. Cryst. Growth 298, 616 (2007).

${ }^{9}$ A. Fontcuberta i Morral, D. Spiroska, J. Arbiol, M. Heigoldt, J. R. Morante, and G. Abstreiter, Small 4, 899 (2008).

${ }^{10}$ F. Glas, Phys. Rev. B 74, 121302 (2006).

${ }^{11}$ F. Glas, J.-C. Harmand, and G. Patriarche, Phys. Rev. Lett. 99, 146101 (2007).

${ }^{12}$ K. A. Dick, P. Caroff, J. Bolinsson, M. E. Messing, J. Johansson, K. Deppert, L. R. Wallenberg, and L. Samuelson, Semicond. Sci. Technol. 25, 024009 (2010).

${ }^{13}$ Y. N. Guo, J. Zou, M. Paladugu, and H. Wang, Appl. Phys. Lett. 89, 231917 (2006).

${ }^{14}$ M. Jeppsson, K. A. Dick, J. B. Wagner, P. Caroff, K. Deppert, L. Samuelson, and L.-E. Wernersson, J. Cryst. Growth 310, 4115 (2008).

${ }^{15}$ D. L. Dheeraj, G. Patriarche, L. Largeau, H. L. Zhou, A. T. J. van Helvoort, F. Glas, J. C. Harmand, B. O. Fimland, and H. Weman, Nanotechnology 19, 275605 (2008).

${ }^{16}$ P. Nimmatoori, Q. Zhang, E. C. Dickey, and J. M. Redwing, Nanotechnology 20, 025607 (2009). 